Andrzej Nowik, University of Warsaw, ul. Banacha 2, 02-097 Warsaw, Poland, email: matan@@paula.univ.gda.pl

\title{
ON A MEASURE WHICH MEASURES AT LEAST ONE SELECTOR FOR EVERY UNCOUNTABLE SUBGROUP
}

\begin{abstract}
We show that there exists in ZFC an invariant extension of Lebesgue measure on $\mathbb{R}$ such that for every uncountable subgroup $H$ of $\mathbb{R}$ there exists at least one selector of $H$ measurable with respect to this measure. This answers a question of Sławomir Solecki in [S].
\end{abstract}

\section{Notation}

Let $H$ be some additive subgroup of $\mathbb{R}$. We denote this fact by $H \leq \mathbb{R}$.

$\operatorname{Symbol} \operatorname{Sel}(H)$ denotes the class of all selectors of the subgroup $H$, i.e., selectors from the class $\{x+H: x \in \mathbb{R}\}$ of cosets of $H$.

Let $B=\left\{h_{\alpha}: \alpha<2^{\omega}\right\}$ be a Hamel base of $\mathbb{R}$. Every $r \in \mathbb{R}$ can be written in the exactly one way as $r=\sum_{\alpha<2^{\omega}} x(\alpha) h_{\alpha}$, where $x(\alpha) \in \mathbb{Q}$ and all $x(\alpha)$ but finitely many are equal to zero. Thus, we can treat $r \in \mathbb{R}$ as such a function $x$. If $x \in \mathbb{R}$ then we put $\operatorname{supp}(x)=\left\{\alpha<2^{\omega}: x(\alpha) \neq 0\right\}$. If $X \subseteq \mathbb{R}$ then $\operatorname{supp}(X)$ denotes $\bigcup_{x \in X} \operatorname{supp}(x)$.

If $X \subseteq \mathbb{R}$ then $\langle X\rangle_{\mathbb{Q}}$ denotes the linear span of $X$ over the field $\mathbb{Q}$. We say that a set $X \subseteq \mathbb{R}$ is totally imperfect set if it does not contain any nonvoid perfect subset of $\mathbb{R}$.

\section{Main Result}

In $[\mathrm{S}]$ the author asked a following question.

Key Words: totally imperfect set, selectors, invariant measures, extensions of measures

Mathematical Reviews subject classification: Primary: 03E05

Received by the editors June 3, 1996

* This paper was supported by the KBN grant 2 P03A 04709. 
Question 1 Does there exist in ZFC an invariant extension of Lebesgue measure on $\mathbb{R}$ such that for every uncountable subgroup $H$ of $\mathbb{R}$ there exists at least one selector of $H$ measurable with respect to this measure?

In $[\mathrm{S}]$ the author proved, that assuming $\mathrm{CH}$ the answer is yes. We will show, that the answer is also positive in ZFC.

Theorem 1 There exists an invariant extension of the Lebesgue measure on $\mathbb{R}$ such that for every uncountable subgroup $H$ of $\mathbb{R}$ there exists at least one selector of $H$ measurable with respect to this measure.

Proof. From [S] we know, that $\mathrm{CH}$ implies Theorem 1. So we consider only the case $\neg \mathrm{CH}$.

Lemma 1 Assume $\neg \mathrm{CH}$. For every subgroup $H \leq \mathbb{R}$ of cardinality $\omega_{1}$ we can find at least one selector $S_{H} \in \operatorname{Sel}(H)$ such that the following condition holds.

$\left(^{*}\right)$ If $\left\{H_{i}\right\}_{i \in \omega}$ is a sequence of subgroups each of power $\omega_{1}$, and $\left\{r_{i}\right\}_{i<\omega}$ are real numbers then $\bigcup_{i=0}^{\infty} S_{H_{i}}+r_{i}$ is not of full (Lebesgue) measure. In fact, this set is totally imperfect.

Proof. Let $F: 2^{\omega} \rightarrow \omega_{1} \times 2^{\omega} \times 2^{\omega}$ be any bijection. We put

$$
F(\alpha)=\left\langle F_{1}(\alpha), F_{2}(\alpha), F_{3}(\alpha)\right\rangle .
$$

Let $\left\{P_{\alpha}\right\}_{\alpha \in 2^{\omega}}$ be an enumeration of all perfect subsets of $\mathbb{R}$. By transfinite induction one can easily construct a Hamel base $B=\left\{h_{\alpha}: \alpha \in 2^{\omega}\right\}$ such that

$$
(\diamond) \quad h_{\alpha} \in P_{F_{2}(\alpha)} \text { for every } \alpha \in 2^{\omega} \text {. }
$$

Take any $H \leq \mathbb{R}$ with $|H|=\omega_{1}$. We construct $S_{H}$ in the following way.

Let $H=\left\{g_{\beta}^{\bar{H}}: \beta<\omega_{1}\right\}$ be an enumeration, without repetitions, of elements of $H$. We take also any selector $T_{H}$ from the class of quotient group $\left\langle\left\{h_{\alpha}: \alpha \in \operatorname{supp}(H)\right\}\right\rangle_{\mathbb{Q}} / H$.

For every $s \in \mathbb{R}$ such that $\operatorname{supp}(s) \cap \operatorname{supp}(H)=\emptyset$ and every $t \in T_{H}$ we put

$$
y_{s, t}:=s+t+g_{F_{1}\left(\alpha_{s}\right)}^{H}
$$

where $\alpha_{s}=\max \operatorname{supp}(s)$. We define $S_{H}$ by:

$$
S_{H}=\left\{y_{s, t}: s \in \mathbb{R} \& \operatorname{supp}(s) \cap \operatorname{supp}(H)=\emptyset \& t \in T_{H}\right\} .
$$

It is easy to see that $S_{H} \in \operatorname{Sel}(H)$. 
Take now any sequence $\left\{H_{i}\right\}_{i \in \omega}$ of subgroups of $\mathbb{R}$ each of power $\omega_{1}$ and a sequence $\left\{x_{i}\right\}_{i \in \omega}$ of real numbers. For each $i \in \omega$ define a number $\beta_{i}<\omega_{1}$ in the following way. Consider $-x_{i} \mid \operatorname{supp}\left(H_{i}\right)$. Obviously $-x_{i} \mid \operatorname{supp}\left(H_{i}\right)=t+g_{\beta}^{H_{i}}$ for some $\beta<\omega_{1}$ and $t \in T_{H_{i}}$ and we define $\beta_{i}$ as this $\beta$.

Let $\beta^{\prime}:=\left(\sup _{i \in \omega} \beta_{i}\right)+1$. Because

$$
\left|\bigcup_{i \in \omega} \operatorname{supp}\left(H_{i}\right) \cup \operatorname{supp}\left(x_{i}\right)\right| \leq \omega_{1}<2^{\omega}
$$

there exists $\alpha \in 2^{\omega}$ such that

$$
(\triangle) \quad \alpha \notin \bigcup_{i \in \omega} \operatorname{supp}\left(H_{i}\right) \quad \text { and } \quad \alpha>\sup _{i \in \omega} \max \operatorname{supp}\left(x_{i}\right)
$$

Let $\gamma^{\prime}=F_{3}(\alpha)$. It is now easy to show, that

$$
h_{\alpha} \notin \bigcup_{i \in \omega} S_{H_{i}}+x_{i} \text { for every } \alpha \text { such that } F_{1}(\alpha)=\beta^{\prime} \text { and } F_{3}(\alpha)=\gamma^{\prime} \text {. }
$$

Indeed, if $h_{\alpha} \in S_{H_{i}}+x_{i}$ for some $i \in \omega$ then

$$
h_{\alpha}-x_{i}\left|\operatorname{supp}\left(H_{i}\right)=-x_{i}\right| \operatorname{supp}\left(H_{i}\right),
$$

because $\alpha \notin \bigcup_{i \in \omega} \operatorname{supp}\left(H_{i}\right)$. So there exists $t \in T_{H}$ such that

$$
-x_{i} \mid \operatorname{supp}\left(H_{i}\right)=t+g_{\beta_{i}}^{H_{i}} .
$$

From $(\triangle)$ we know that $\max \operatorname{supp}\left(h_{\alpha}-x_{i}\right)=\alpha$. Therefore if $s=\left(h_{\alpha}-\right.$ $\left.x_{i}\right) \mid\left(2^{\omega} \backslash \operatorname{supp}\left(H_{i}\right)\right)$ then $y_{s, t}=s+t+g_{\beta^{\prime}}^{H_{i}}$ from the definition of $y_{s, t}$. But $h_{\alpha}-x_{i}=\left(h_{\alpha}-x_{i}\right) \mid\left(2^{\omega} \backslash \operatorname{supp}\left(H_{i}\right)\right)+t+g_{\beta_{i}}^{H_{i}}$, since $\alpha>\max \operatorname{supp}\left(x_{i}\right)$ and $\beta^{\prime}=F(\alpha)$. So $g_{\beta_{i}}^{H_{i}}=g_{\beta^{\prime}}^{H_{i}}$, a contradiction with the definition of $\beta^{\prime}$, finishing the proof of (1).

Now $h_{\alpha} \notin \bigcup_{i \in \omega} S_{H_{i}}+x_{i}$ for every $\alpha \in 2^{\omega}$ such that $F_{1}(\alpha)=\beta^{\prime}$ and $F_{3}(\alpha)=\gamma^{\prime}$, and because $h_{\alpha} \in P_{F_{2}(\alpha)}$, we obtain that for every $\delta \in 2^{\omega}$,

$$
P_{\delta} \backslash \bigcup_{i \in \omega}\left(S_{H_{i}}+x_{i}\right) \neq \emptyset \text {. }
$$

Therefore the set

$$
\bigcup_{i \in \omega} S_{H_{i}}+x_{i}
$$

is not of full measure. In fact, this set is totally imperfect.

In the next part of the proof of Theorem 1 we will use the following Lemma from $[\mathrm{S}]$. 
Lemma $2[S z]$ Let $\mu$ be a translation invariant measure on $\mathbb{R}$ and let $J$ be an invariant $\sigma$-ideal on $\mathbb{R}$ such that $\mu_{*}(A)=0$ for $A \in J$ (where $\mu_{*}$ denotes the inner measure induced by $\mu$ ). Then there exists an invariant extension of $\mu$ defined on the $\sigma$-field generated by the union of the $\sigma$-field of $\mu$ measurable subsets of $\mathbb{R}$ and $J$.

Using now Lemma 1 and Lemma 2 we easily obtain our extension.

In fact, let $J$ be the $\sigma$-ideal generated by all $S_{H}+x$, where $x \in \mathbb{R}, H \leq \mathbb{R}$, $|H|=\omega_{1}$. Assume by way of contradiction, that $\mu_{*}(A)>0$ for some $A \in J$, where $\mu$ is Lebesgue measure. Using the Steinhaus theorem for the Lebesgue measure we obtain that $\mathbb{Q}+A$ is of full measure. But $\mathbb{Q}+A \in J$, which gives contradiction with Lemma 1.

Using Lemma 2 we obtain, that there exists an invariant extension $\bar{\mu}$ of Lebesgue measure such that every set from $J$ is measurable with respect to

$\bar{\mu}$, so for every uncountable subgroup of $\mathbb{R}$ there exists at least one selector measurable with respect to $\bar{\mu}$.

\section{References}

[S] S. Solecki, Measurability properties of sets of Vitali's type, Proc. Amer. Math. Soc. 119, (1993) 897-902.

[Sz] E. Szpilrajn (Marczewski), Sur l'extension de la mesure lebesguienne, Fund. Math. 25, (1935) 551-558. 